\title{
Um Jogo Sério em 3D para Apoiar a Aprendizagem Sensível ao Contexto dos Estudantes
}

\author{
Rodrigo Monteiro de Lima, Francisco Milton Mendes Neto, Rafael de Almeida \\ Rodrigues
}

Bacharelado em Ciência da Computação, Departamento de Ciências Exatas e Naturais, Universidade Federal Rural do Semi-Árido (UFERSA) - BR 110 - Km 47 - 59625-900

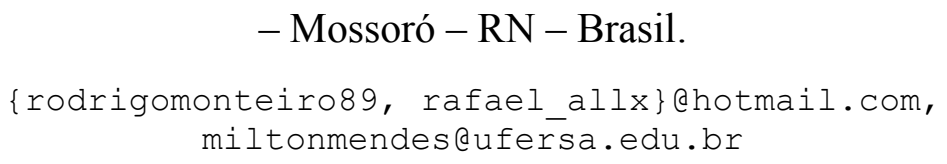

\begin{abstract}
This paper describes the development of an ubiquitous learning game capable of simulating a virtual university for mobile devices and desktops. In the case of mobile devices, the serious game is able to sense both the location and the environment of users in order to recommend related challenges to location and/or area of study. The user will also learn various concepts of disciplines to interact with avatars present in the virtual environment. The virtual environment also has challenges using augmented reality for the player to better assimilate the concepts learned in the classroom through real world applications.
\end{abstract}

Resumo. Este artigo descreve o desenvolvimento de um jogo de aprendizagem ubíqua capaz de simular uma universidade virtual para dispositivos móveis, bem como desktops. No caso dos dispositivos móveis, o jogo sério é capaz de perceber a localização do usuário e identificar em qual ambiente o usuário se encontra, para, posteriormente, recomendar desafios relacionados àquela localidade ou área de estudo. O usuário também aprenderá diversos conceitos de disciplinas ao interagir com avatares presentes no ambiente virtual. $O$ ambiente virtual também conta com desafios que utilizam realidade aumentada para que o jogador assimile melhor os conceitos aprendidos em sala de aula através de aplicações do mundo real.

\section{Introdução}

Atualmente professores utilizam recursos adicionais para simular fenômenos que estão sendo estudados em sala de aula. No entanto, esta prática normalmente se limita à demonstração de fenômenos apresentados pelo professor. $\mathrm{O}$ aluno continua participando apenas como expectador e não tem a oportunidade de realizar experimentos diversos e individuais. A aula, com o auxílio desses recursos, continua sendo expositiva e os alunos se mantêm em uma posição passiva no processo de aquisição de conhecimento. Quando alunos têm a oportunidade de realizar experimentos próprios, eles passam a assumir uma postura ativa no processo de aprendizagem e constroem o conhecimento necessário. 
A fim de facilitar esse processo de ensino-aprendizagem, foi criado, no cenário de uma Universidade Virtual, um jogo sensível ao contexto dos estudantes com desafios educativos em 2D e 3D para diversos dispositivos computacionais, pois funciona na web precisando apenas de um navegador de internet para ser executado.

Este artigo está organizado da seguinte forma: na Seção 2, são apresentados alguns trabalhos relacionados à proposta. Na Seção 3, são apresentados os temas aprendizagem móvel, aprendizagem ubíqua e jogos sérios. Na Seção 4, são mostradas algumas interfaces da aplicação, uma descrição do ambiente e o mecanismo de recomendação utilizado. Por fim, a Seção 5 traz nossas considerações finais e perspectivas de trabalhos futuros.

\section{Trabalhos relacionados}

O Second Life [Rosendale 2012], desenvolvido a partir de 2003 pela empresa norte-americana Linde Lab, é um mundo virtual imersivo 3D onde seus habitantes interagem com avatares, objetos e conteúdos. Tem sido utilizado com sucesso na criação de várias universidades virtuais. No entanto, o Second Life também apresenta alguns pontos negativos, sendo o mais forte deles a questão de não ser um software livre. Por isso, embora os seus muitos usuários possam navegar e interagir uns com os outros de forma inteiramente livre, uma instituição educacional que tenha intenção de utilizar esse ambiente em suas atividades didáticas deve locar um espaço, sendo que tal espaço deve ser mantido mensalmente.

O Open Wonderland [Yankelovich 2012] é um mundo virtual imersivo baseado em Java com um motor gráfico que gerencia o ambiente, as animações e os avatares presentes no mundo virtual. Além disso, ele suporta o compartilhamento de aplicativos, bem como mundos extensíveis e personalizáveis. A Figura 1 apresenta alguns usuários interagindo entre si no ambiente virtual.

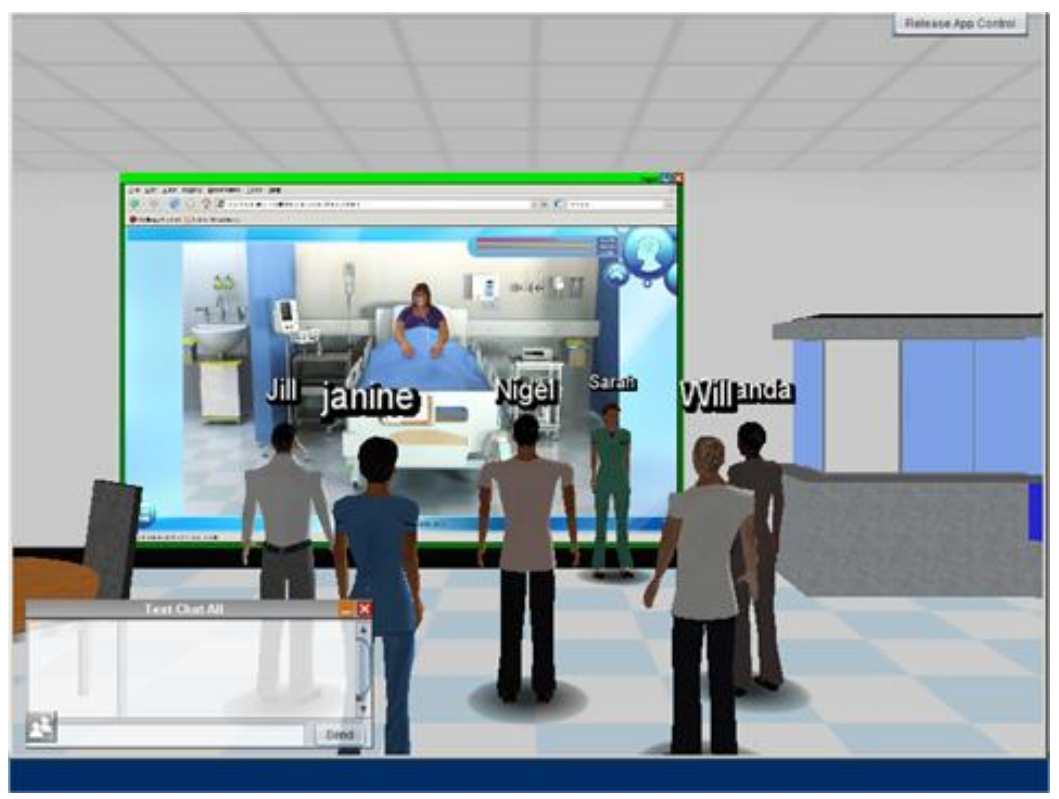

Figura 1. Open Wonderland

É importante frisar que o Open Wonderland e o Second Life, embora possuam objetivos comuns, estão em níveis de desenvolvimento diferentes. Enquanto o Second Life já é utilizado como produto e acessado por milhões de usuários, o Open Wonderland ainda está em processo 
de desenvolvimento, sendo utilizado em grande parte por programadores e acadêmicos. Sendo este último um mundo virtual que possui código aberto [Cunha e Mainente 2011].

Diversos softwares brasileiros também foram criados nos últimos anos a fim de facilitar o processo de ensino-aprendizagem nas instituições de ensino. Alguns exemplos são o MyQuímica [Silva e Rebouças 2011] e o ProGame [Dantas e Alencar 2011]. O MyQuímica é um jogo sério desenvolvido para dispositivos móveis com o sistema operacional Android. Este jogo tem como finalidade familiarizar alunos com a tabela periódica e com as nomenclaturas químicas, o jogo propõe aos alunos diversos desafios que consistem em arrastar de uma tabela periódica para um tubo de ensaio os elementos de um certo composto químico cuja nomenclatura é apresentada. Já o ProGame é um jogo sério criado para apoiar o aprendizado de programação de forma lúdica, dinâmica e interativa e, ao mesmo tempo, motivar os alunos a aprenderem, combatendo os altos índices de evasão e reprovação da disciplina. O ProGame é um jogo de aventura que insere o jogador em diversos cenários, propondo desafios que estimulam o jogador a praticar seus conhecimentos de programação nas linguagens Python ou Java.

\section{Aprendizagem móvel, aprendizagem ubíqua e jogos sérios}

Os avanços das tecnologias móveis e de comunicação sem fio também contribuíram para demarcar um novo horizonte aos processos de ensino e aprendizagem já estabelecidos. Os dispositivos móveis possibilitam o acesso a ambientes virtuais de ensino e aprendizagem, caracterizando, assim, a aprendizagem móvel, do inglês Mobile Learning ou m-learning [Saccol et al. 2010]. Este meio de oferecer ensino permite que estudantes e professores possam tirar vantagens dos recursos oferecidos pelas tecnologias móveis, dentre as quais se destaca a possibilidade de acessar, visualizar e prover conteúdo independentemente do horário e a partir de qualquer localidade [Yau e Joy 2010].

Entretanto, as tecnologias móveis promovem o acesso a uma ampla quantidade e diversidade de conteúdo educacional, o que pode acarretar em perda de desempenho dos estudantes, por provocar a dispersão destes. Para lidar com essa situação, ao fornecer conteúdo educacional, deve-se levar em consideração as características particulares de cada estudante. Surge então o conceito de ambientes sensíveis ao contexto (context-aware environments). Esse tipo de ambiente se adapta ao perfil do usuário, levando em consideração informações fornecidas pelo próprio usuário, além daquelas captadas dinamicamente a partir de sua interação com os dispositivos computacionais [Moore et al. 2009].

Outra tecnologia que tem surgido como meio atrativo e motivador de ensino são os chamados jogos educativos (educational games) ou jogos sérios (serious games). O aprendizado baseado em jogos educativos ou jogos sérios está se tornando uma tendência importante na área de pesquisa em Informática na Educação, pois trata de vários problemas típicos do Ensino a Distância (EaD), como altas taxas de abandono devido à frustração e à falta de motivação para continuar a estudar e a sobrecarga cognitiva do aluno [Yessad et al. 2010].

Essa nova abordagem de jogos educativos eletrônicos a distância tem emergido como um meio mais atrativo e motivador de $\mathrm{EaD}$ e é uma das áreas mais promissoras para a melhoria da educação [Junqi et al. 2009]. Apesar desse crescimento, o uso de jogos para a aprendizagem é discutível, pois são geralmente vistos como instrumentos para diversão [Hodgson et al. 2010]. No entanto, como o jogo requer dos jogadores concentração e dedicação para superar os 
desafios, os professores podem usar essa motivação para permitir aquisição de conhecimento e habilidades por parte dos alunos. Estudos indicam que jogadores melhoram sua concentração, seus reflexos e sua capacidade de tomada de decisões rápidas [Morsi e Jackson 2007].

\section{Um jogo virtual 3D simulando uma universidade real}

Nesta seção serão apresentadas as principais características do jogo sério em 3D para apoiar a aprendizagem sensível ao contexto dos estudantes, que simula uma universidade virtual para dispositivos móveis, proposto neste trabalho. A Subseção 4.1 apresenta algumas interfaces do jogo e um cenário de uso. A Subseção 4.2 descreve o mecanismo de recomendação utilizado. Por fim, a Subseção 4.3 apresenta sua arquitetura física e aspectos de implementação.

\subsection{Cenários de uso e interfaces do jogo sério}

Inicialmente, o estudante cadastra-se no sistema inserindo seus dados na página de cadastro. Após o cadastro ser efetuado com sucesso, o estudante pode usar o login e a senha cadastrados no sistema para entrar no jogo. Dentro do ambiente, o usuário pode escolher as opções Perfil, Amigos, Universidade Virtual, Chat, Notícias e Geolocalização.

Na opção Perfil, o usuário visualiza seus dados, tais como nome, sobrenome, instituição de ensino, cidade, país, área(s) de afinidade (ex.: informática, medicina, biologia, etc.), melhor horário de estudo e uma foto do usuário. Na opção Amigos, o estudante é informado sobre sua lista de amizades. Ainda nesta opção, o estudante visualiza todos os usuários cadastrados no sistema com afinidades semelhantes. Na opção Universidade Virtual, o estudante pode interagir com os avatares presentes no ambiente ou tentar solucionar (jogando) os desafios propostos nos laboratórios espalhados pelo jogo. A Figura 2 apresenta o jogo sendo carregado dentro da opção Universidade Virtual.

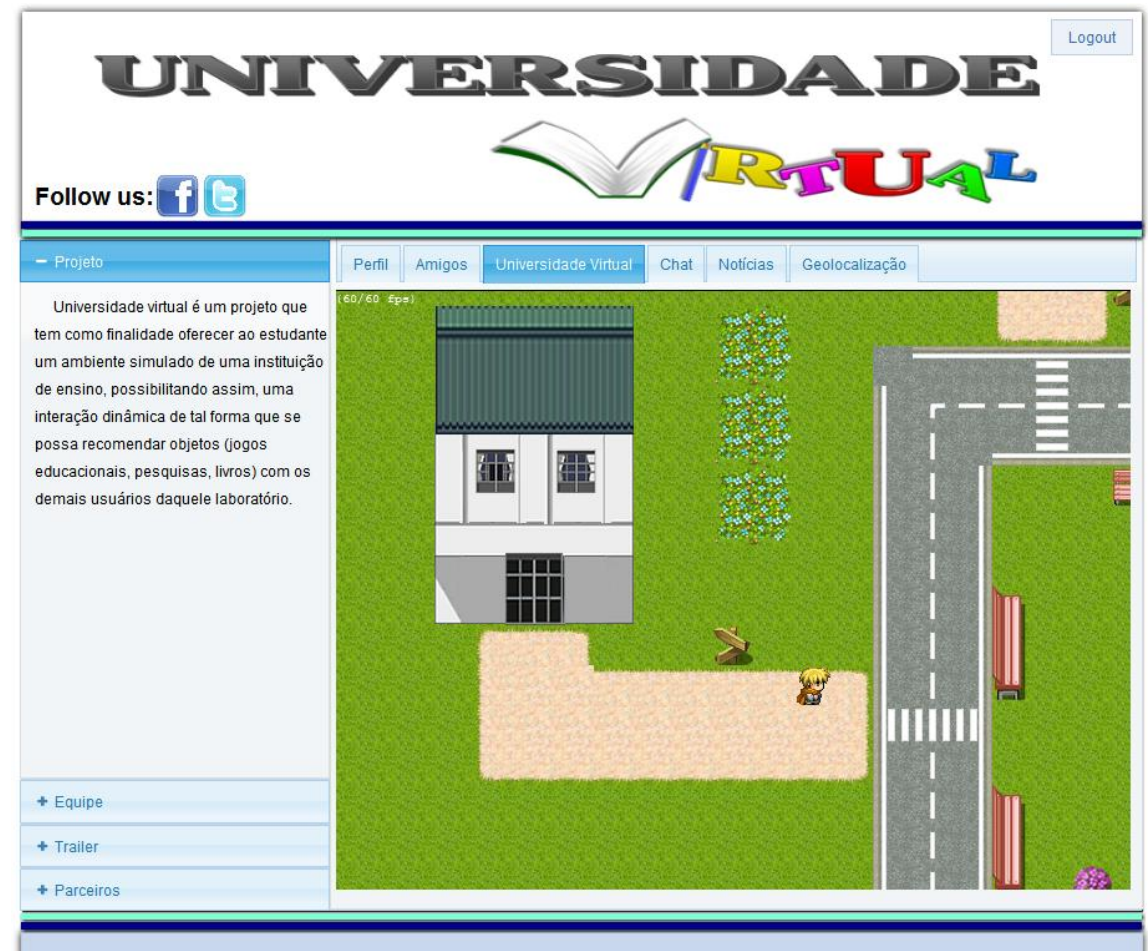

Figura 2. Interface principal aberta com a opção do jogo selecionada 
Na Figura 3(a), tem-se um exemplo do laboratório de física e, na Figura 3(b), um exemplo do laboratório de engenharia de petróleo, em 3D.

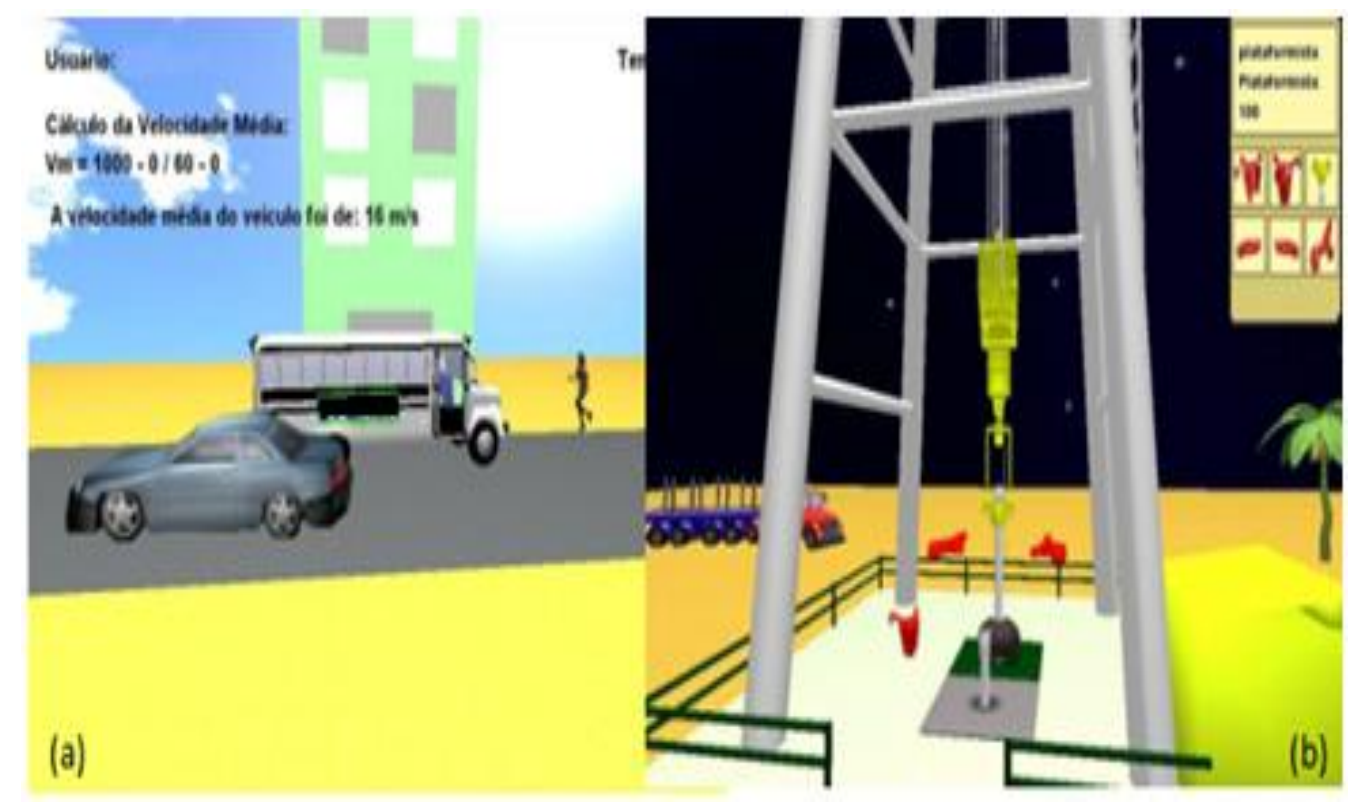

Figura 3. (a) Exemplo de jogo 3D no cálculo da velocidade média (b) Exemplo de jogo 3D na perfuração de um poço de petróleo terrestre

$\mathrm{Na}$ opção Chat, o estudante pode conversar com todos os amigos cadastrados em sua lista de amizade. Pode também conhecer pessoas com a mesma área de afinidade, melhorando seu convívio social. Escolhendo a opção Notícias, o mesmo visualiza as últimas notícias cadastradas pelos professores/administradores. Estas notícias podem ser novas opções no ambiente, atualizações, novos desafios propostos, etc.

O estudante pode usar as opções de Criar novo local e Check In, presentes na opção geolocalização. Na opção Criar novo local, o servidor guarda a latitude e a longitude, que podem ser capturadas automaticamente pelo dispositivo móvel, além de outras características do local cadastrado. Na opção de Check In, o dispositivo móvel do estudante passa, automaticamente, para o servidor suas atuais coordenadas, a fim de determinar o lugar onde este se encontra. A partir do momento que o estudante estiver em um local cadastrado no sistema, o servidor recomenda desafios ou assuntos relacionados ao contexto do estudante. Por exemplo, se um estudante estiver no laboratório de química de sua universidade, ele receberá desafios e assuntos relacionados à disciplina de química. As pontuações adquiridas pelo estudante naquele jogo ficam armazenadas no servidor de banco de dados, da mesma forma como ocorre com os desafios resolvidos. Ao final de cada desafio, a pontuação é cadastrada no servidor para posteriores consultas e aplicações. A Figura 4 mostra a opção Geolocalização selecionada pelo usuário e sua localização atual. 


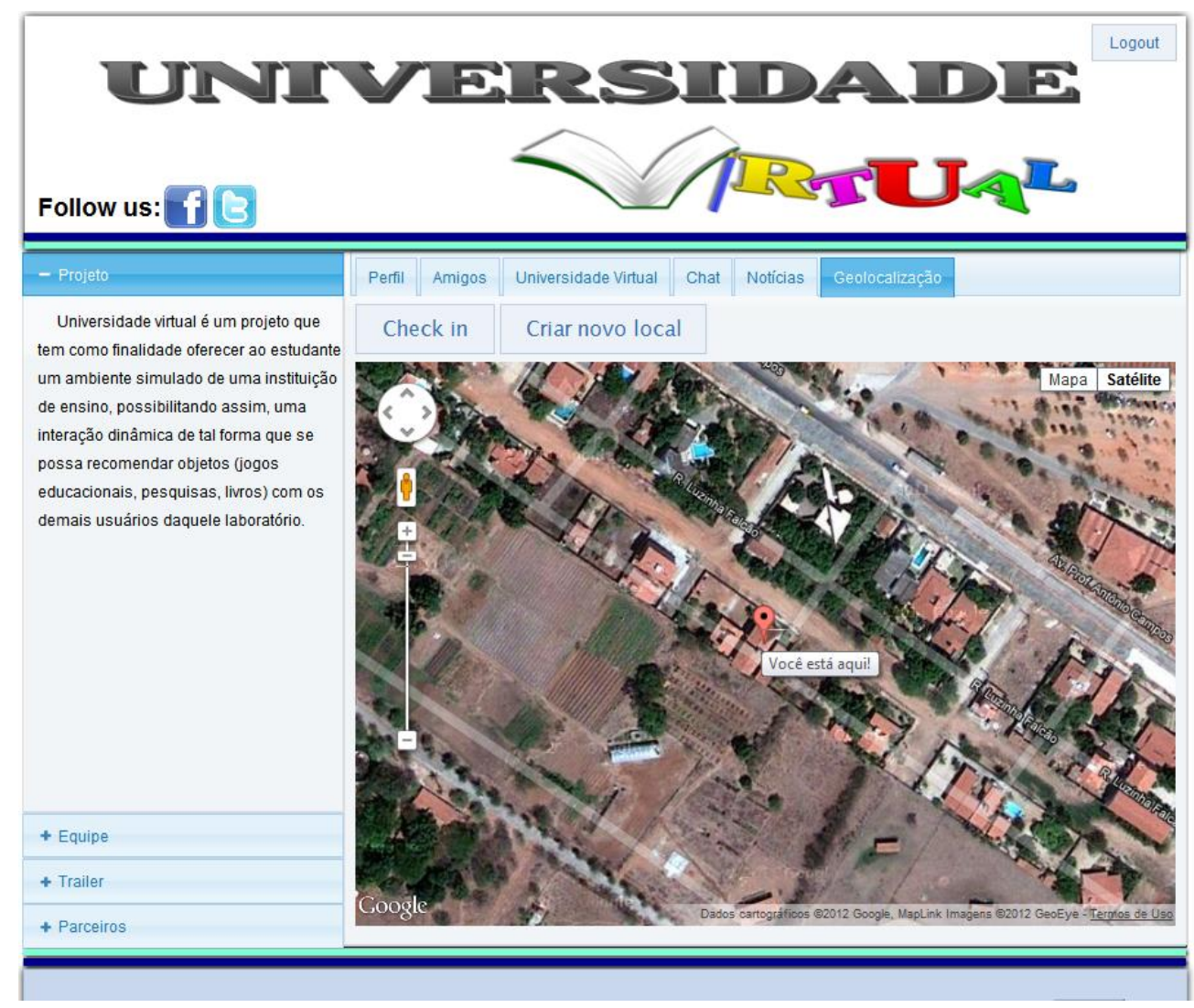

Figura 4. Opção de geolocalização selecionada mostrando a atual localização do usuário

\subsection{Mecanismo de Recomendação}

A opção de Check In, presente na opção Geolocalização, não funciona caso o usuário não a selecione. Utilizando-se do GPS presente em dispositivos móveis, criou-se um programa que envia periodicamente informações ao servidor, comparando-as com dados dos estudantes armazenados na base de dados. Esse mecanismo de recomendação funciona em segundo plano, ficando transparente ao usuário, entrando em ação apenas quando detecta situações ideais para recomendação de desafios ou assuntos.

O mecanismo de recomendação considera as informações de localidade cadastradas, tais como nome do local, longitude e latitude, como também informações relacionadas à área de interesse do estudante e horário preferido de estudo. Caso as informações comparadas estejam de acordo com o perfil do estudante, desafios e assuntos de interesse são enviados para o mesmo, através de uma aplicação que é executada em segundo plano no dispositivo móvel. Para alertá-lo de novas recomendações, o ambiente emite um som. Na Figura 5, é mostrado um exemplo do mecanismo de recomendação. 


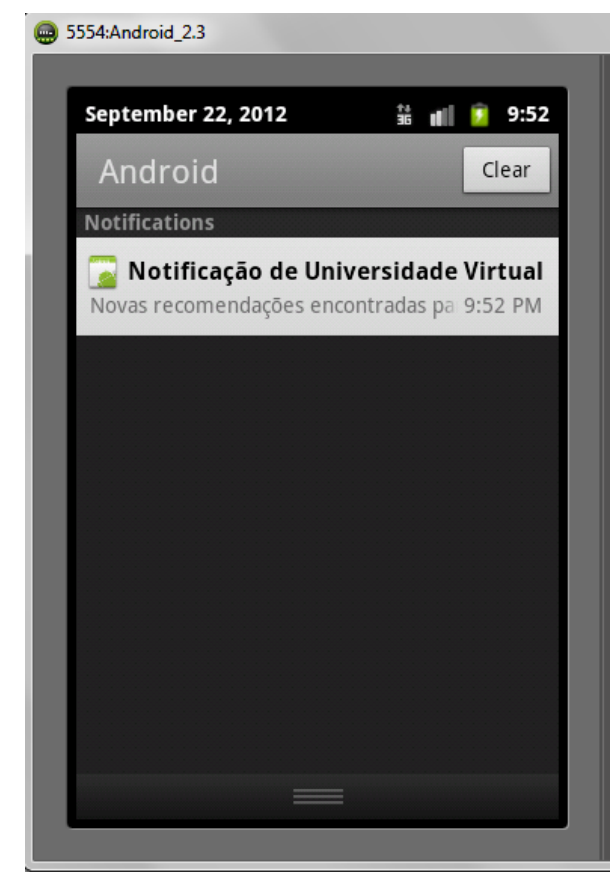

\section{Figura 5. Estudante recebendo uma notificação com novas recomendações encontradas}

Algumas recomendações podem utilizar realidade aumentada para facilitar o processo de aprendizagem do aluno. Para que a realidade aumentada seja executada com sucesso, basta o aluno clicar sobre a nova recomendação, isso fará com que ele seja encaminhado para um programa na internet que acionará sua webcam. Feito isso, basta apenas que o estudante capture, através da webcam de seu dispositivo, uma imagem enviada para o seu respectivo email, fazendo com que o objeto $3 \mathrm{D}$ seja mostrado na tela. $\mathrm{O}$ sistema então exibe desafios ou conteúdos relacionados àquele objeto. Como pode ser visto no exemplo apresentado na Figura 6 , a imagem sendo mostrada pelo celular é capturada pela webcam do computador que executa a realidade aumentada.

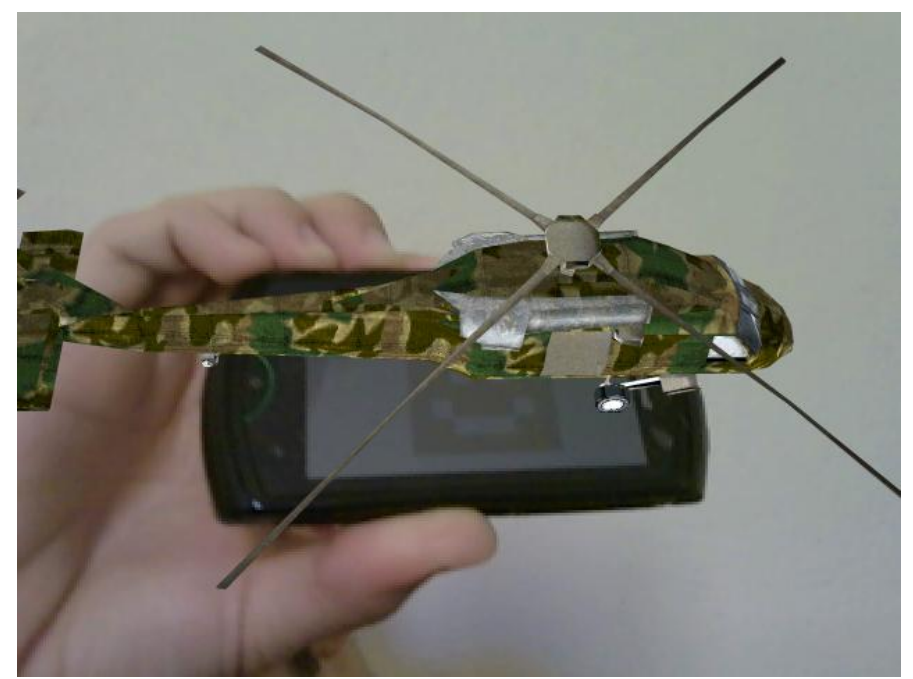

Figura 6. Exemplo de uma recomendação contendo realidade aumentada

\subsection{Aspectos de implementação}


O ambiente proposto foi desenvolvido com a nova linguagem de marcação HTML5 [Silva 2011] para organização e formatação das interfaces do sistema. Trabalhando junto ao HTML5, tem-se o JavaScript [Silva 2010] para criação de funções no lado do cliente. Dessa maneira, alguns processamentos desnecessários são evitados no lado do cliente, a fim de aumentar a usabilidade e velocidade do sistema. Na Figura 7, é apresentada a arquitetura física do sistema.

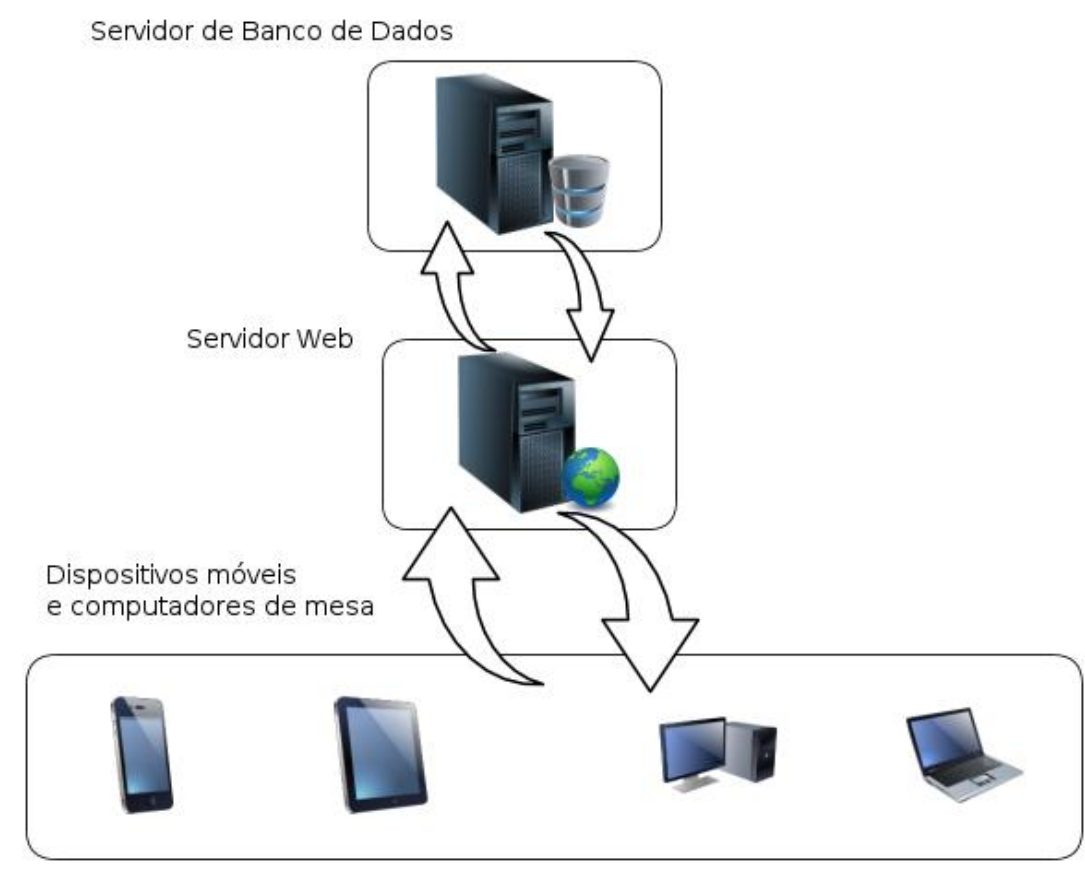

Figura 7. Arquitetura física do ambiente

Para criação do mapa do ambiente 2D, usou-se a ferramenta Tiled Map Editor [Lindeijer 2012]. A principal característica desta ferramenta é que ela cria mapas baseados em arquivos XML (eXtensible Markup Language) [Quin 2012], o que possibilita sua manipulação junto com a biblioteca MelonJS [Olivier 2012]. Essa biblioteca consiste em um conjunto de funções em JavaScript para manipulação e criação de jogos em 2D. Por ser em JavaScript, esta biblioteca possibilita a criação de um ambiente na web juntamente com o HTML5.

Para a criação dos jogos 3D para dispositivos móveis e computadores de mesa, foram usadas as bibliotecas JPCT-AE e JPCT [Olsen 2012], respectivamente.

Para a criação das aplicações usando realidade aumentada, foi usado o programa EZFLAR [Malpeli et al. 2012], desenvolvido por técnicos do jornal O Estado de São Paulo. O programa possui código aberto e pode ser usado e melhorado por programadores. Como prérequisito de qualquer aplicação que use a tecnologia de realidade aumentada, os jogos criados só funcionam em dispositivos móveis que possuam câmera digital integrada.

Para a parte lógica de busca, inserção, remoção e edição de usuários na base de dados e de outros dados, foi utilizada a linguagem PHP (acrônimo de Hypertext Preprocessor) e o $S G B D$ (Sistema de Gerenciamento de Banco de Dados) MySQL [Milani 2010].

\section{Considerações finais e trabalhos futuros}

A partir de estudos realizados sobre trabalhos relacionados nesta área, não foi encontrado nenhum ambiente 3D de aprendizagem que auxiliasse o ensino de diversas áreas de maneira 
lúdica e interativa para dispositivos móveis e computadores de mesa usando conceitos de aprendizagem ubíqua. $\mathrm{O}$ ambiente proposto no presente trabalho tira proveito das mais recentes tecnologias de apoio à educação, tais como aprendizagem sensível ao contexto, jogos sérios e aprendizagem móvel, a fim de criar um ambiente atrativo, motivador ao aluno, acessado de qualquer lugar e adaptado ao contexto dos estudantes.

Como trabalhos futuros, serão criados agentes inteligentes que controlarão os avatares, a fim de deixar o ambiente o mais real e diversificado possível. Outra característica que será incorporada no jogo é o ambiente ser multijogador, através da tecnologia websockets [Hickson 2012], projetada para comunicação bidirecional em browsers e servidores web que suportem o HTML5. O estudante poderá jogar em qualquer dispositivo computacional, seja ele móvel ou não, interagindo com todos os usuários on-line no ambiente.

Também será incorporada a Teoria da Carga Cognitiva [Araújo e Rockenbach 2007] ao ambiente proposto. Assim, os desafios impostos pelo ambiente serão adaptados à forma como o estudante se saiu em tentativas anteriores. Por exemplo, caso o estudante tenha se saído mal com um desafio, este desafio, ao ser acessado posteriormente, apresentará um grau de dificuldade menor do que o anterior, requerendo uma menor sobrecarga cognitiva por parte do jogador. Caso o jogador tenha se saído bem, a questão ficará mais desafiadora, fazendo com que o estudante precise de um maior raciocínio para a resolução daquele desafio.

\section{Referências}

Araújo, S. L. M. e Rockenbach, T. L. M. (2007) "A Importância do Estudo da Teoria da Carga Cognitiva em uma Educação Tecnológica”. Revista Novas Tecnologias na Educação vol. 5 no.1, Rio Grande do Sul, Julho de 2007.

Cunha, F. M. T e Mainente, A. C. (2011) "Utilização de ambientes virtuais 3D no ensino de ciência da computação: estado da arte". São Caetano do Sul, Maio de 2011.

Dantas, V. F.; Alencar, L. A. (2011) "ProGame: Um Jogo para Apoiar o Ensino-Aprendizagem de Programação". Aracaju-SE, Novembro de 2011. $22^{\circ}$ Simpósio Brasileiro de Informática na Educação e $17^{\circ}$ Workshop de Informática na Escola. ISSN: 2176-4301.

Hickson, I. (2012) "WebSockets". Disponível em: www.websocket.org. Acessado em 06/09/2012.

Hodgson, P.; Man, D.; Leung, J. Managing the development of digital educational games. In: Proceedings of the 2010 Third IEEE International Conference on Digital Game and Intelligent T oy Enhanced Learning. Washington, DC, USA: IEEE Computer Society, 2010. (DIGITEL '10), p. 191-193. ISBN 978-0-7695-3993-5. Disponível em: $<$ http://dx.doi.org/10.1109/DIGITEL.2010.18>.

Junqi, W. et al. Strategies for designing educational computer game. In: Education Technology and Computer Science, 2009. ETCS '09. First International Workshop on. [S.1.: s.n.], 2009. v. 3, p. $950-952$.

Lindeijer, T. (2012) “Tiled Map Editor”. Disponível em: www.mapeditor.org, Acessado em $06 / 09 / 2012$.

Malpeli, E.; Roda, D.; Freitas, A. (2012) “Ezflar”. Disponível em: www.ezflar.com, Acessado em 06/09/2012. 
Milani, A. (2010) "Contruindo Aplicações Web com PHP e MySQL” $1^{\circ}$ Edição, pág. 40-200. Editora Novatec.

Moore, P. et al. Intelligent Context for Personalised M -Learning. International Conference on Complex, Intelligent and Software Intensive Systems (CISIS). Birmingham: Birmingham City University. 2009. p. 247 -254.

Morsi, R.; Jackson, E. Playing and learning? Educational gaming for engineering education. In: Frontiers In Education Conference - Global Engineering: Knowledge Without Borders, Opportunities Without Passports, 2007. FIE '07. 37th Annual. [S.1.: s.n.], 2007. p. F2H-1F2H-6. ISSN 0190-5848.

Olivier, B. (2012) “melonJS”. Disponível em: www.melonjs.org, Acessado em 06/09/2012.

Olsen, E. (2012) “JPCT e JPCT-AE”. Disponível em: www.jpct.net, Acessado em 06/09/2012.

Quin, L. (2012) "XML eXtensible Markup Language”. Disponível em: www.w3.org/XML, Acessado em 06/09/2012.

Rosendale, P. (2012) "Second Life". Disponível em: www.secondlife.com, Acessado em 06/09/2012.

Saccol, A., Schlemmer, E., Barbosa, J. m-learning e u-learning - Novas perspectivas da aprendizagem móvel e ubíqua. São Paulo, SP: Pearson, 2010.

Silva, S. M. (2010) “JavaScript Guia do Programador”. $1^{\circ}$ Edição, pág. 20-30. Editora Novatec.

Silva, S. M. (2011) "HTML5 A Linguagem de Marcação que Revolucionou a Web". $1^{\circ}$ Edição, pág. 20-52. Editora Novatec.

Silva, D. L. M.; Rebouças, A. D. D. (2011) "MyQuímica - Um Jogo para Auxiliar no EnsinoAprendizagem das nomenclaturas Químicas". Aracaju-SE, Novembro de 2011. $22^{\circ}$ Simpósio Brasileiro de Informática na Educação e $17^{\circ}$ Workshop de Informática na Escola. ISSN: 2176-4301.

Yankelovich, N. (2012) “Open Wonderland”. Disponível em: www.openwonderland.org, Acessado em 06/09/2012.

Yau, J. Y.-K.; Joy, M. A Context-Aware Personalized M-learning Application Based on M learning Preferences. 6th IEEE International Conference on Wireless, Mobile and Ubiquitous Technologies in Education (WMUTE). Reino Unido: IEEE Computer Society. 2010. p. 11-18.

Yessad, A.; Labat, J. M.; Kermorvant, F. Segae: A serious game authoring environment. In: Proceedings of the 2010 10th IEEE International Conference on Advanced Learning Technologies. Washington, DC, USA: IEEE Computer Society, 2010. (ICALT '10), p. 538540. ISBN 978-0-7695-4055-9. Disponível em: http://dx.doi.org/10.1109/ICALT.2010.153. 